\title{
BMJ Open SAFety, Effectiveness of care and Resource use among Australian Hospitals (SAFER Hospitals): a protocol for a population-wide cohort study of outcomes of hospital care
}

\author{
Isuru Ranasinghe (D) , ${ }^{1,2,3}$ Sadia Hossain, ${ }^{3}$ Anna Ali, ${ }^{3}$ Dennis Horton, ${ }^{3}$ \\ Robert JT Adams, ${ }^{3,4}$ Bernadette Aliprandi-Costa, ${ }^{5}$ Christina Bertilone, ${ }^{6}$ \\ Gustavo Carneiro, ${ }^{7}$ Martin Gallagher, ${ }^{8,9}$ Steven Guthridge, ${ }^{10}$ Billingsley Kaambwa, ${ }^{11}$ \\ Sradha Kotwal, ${ }^{8,12}$ Gerry O'Callaghan, ${ }^{3,13}$ Ian A Scott (D) ,2,14 \\ Renuka Visvanathan, ${ }^{3,15,16}$ Richard J Woodman ${ }^{17}$
}

To cite: Ranasinghe I, Hossain S, Ali A, et al. SAFety, Effectiveness of care and Resource use among Australian Hospitals (SAFER Hospitals): a protocol for a population-wide cohort study of outcomes of hospital care. BMJ Open 2020;10:e035446. doi:10.1136/ bmjopen-2019-035446

- Prepublication history and additional material for this paper are available online. To view these files, please visit the journal online (http://dx.doi org/10.1136/bmjopen-2019035446).

Received 01 November 2019 Revised 05 May 2020 Accepted 09 July 2020

D) Check for updates

(c) Author(s) (or their employer(s)) 2020. Re-use permitted under CC BY-NC. No commercial re-use. See rights and permissions. Published by BMJ.

For numbered affiliations see end of article.

Correspondence to Associate Professor Isuru Ranasinghe;

i.ranasinghe@uq.edu.au

\section{ABSTRACT}

Introduction Despite global concerns about the safety and quality of health care, population-wide studies of hospital outcomes are uncommon. The SAFety, Effectiveness of care and Resource use among Australian Hospitals (SAFER Hospitals) study seeks to estimate the incidence of serious adverse events, mortality, unplanned rehospitalisations and direct costs following hospital encounters using nationwide data, and to assess the variation and trends in these outcomes.

Methods and analysis SAFER Hospitals is a cohort study with retrospective and prospective components. The retrospective component uses data from 2012 to 2018 on all hospitalised patients age $\geq 18$ years included in each State and Territories' Admitted Patient Collections. These routinely collected datasets record every hospital encounter from all public and most private hospitals using a standardised set of variables including patient demographics, primary and secondary diagnoses, procedures and patient status at discharge. The study outcomes are deaths, adverse events, readmissions and emergency care visits. Hospitalisation data will be linked to subsequent hospitalisations and each region's Emergency Department Data Collections and Death Registries to assess readmissions, emergency care encounters and deaths after discharge. Direct hospital costs associated with adverse outcomes will be estimated using data from the National Cost Data Collection. Variation in these outcomes among hospitals will be assessed adjusting for differences in hospitals' case-mix. The prospective component of the study will evaluate the temporal change in outcomes every 4 years from 2019 until 2030.

Ethics and dissemination Human Research Ethics Committees of the respective Australian states and territories provided ethical approval to conduct this study. A waiver of informed consent was granted for the use of de-identified patient data. Study findings will be disseminated via presentations at conferences and publications in peer-reviewed journals.
Strengths and limitations of this study

- SAFety, Effectiveness of care and Resource use among Australian Hospitals is a population-wide study that uses routinely collected administrative data from all public and most (86\%) private hospitals in Australia to estimate the national incidence of serious adverse events, deaths and unplanned hospitalisations, and measure how these outcomes vary among hospitals.

- It will also use publicly available summary cost data to estimate the avoidable direct healthcare costs associated with adverse outcomes of care.

- It has retrospective and prospective components that will enable monitoring of temporal change in patient characteristics and outcomes over time.

- The major strength of this study is comprehensive data linkage enabling reporting of outcomes at a national scale including patient outcomes after discharge.

- The main limitation of the study is unmeasured confounding from the use of routinely collected administrative data which is less granular than data collected solely for research, although a populationwide study on this scale would not be feasible without using administrative data.

\section{INTRODUCTION}

Modern hospital care is fast-paced, complex and expensive. While this care has undoubtedly led to advances in care for many patients, the Institute of Medicine report 'To Err is Human' highlighted an uncomfortable truth-modern care also leads to considerable harm with $\sim 98000$ patient deaths attributed to medical error. ${ }^{1}$ In addition to deaths, patients experience high rates of adverse events, with a systematic review of pivotal studies ${ }^{2}$ from the 


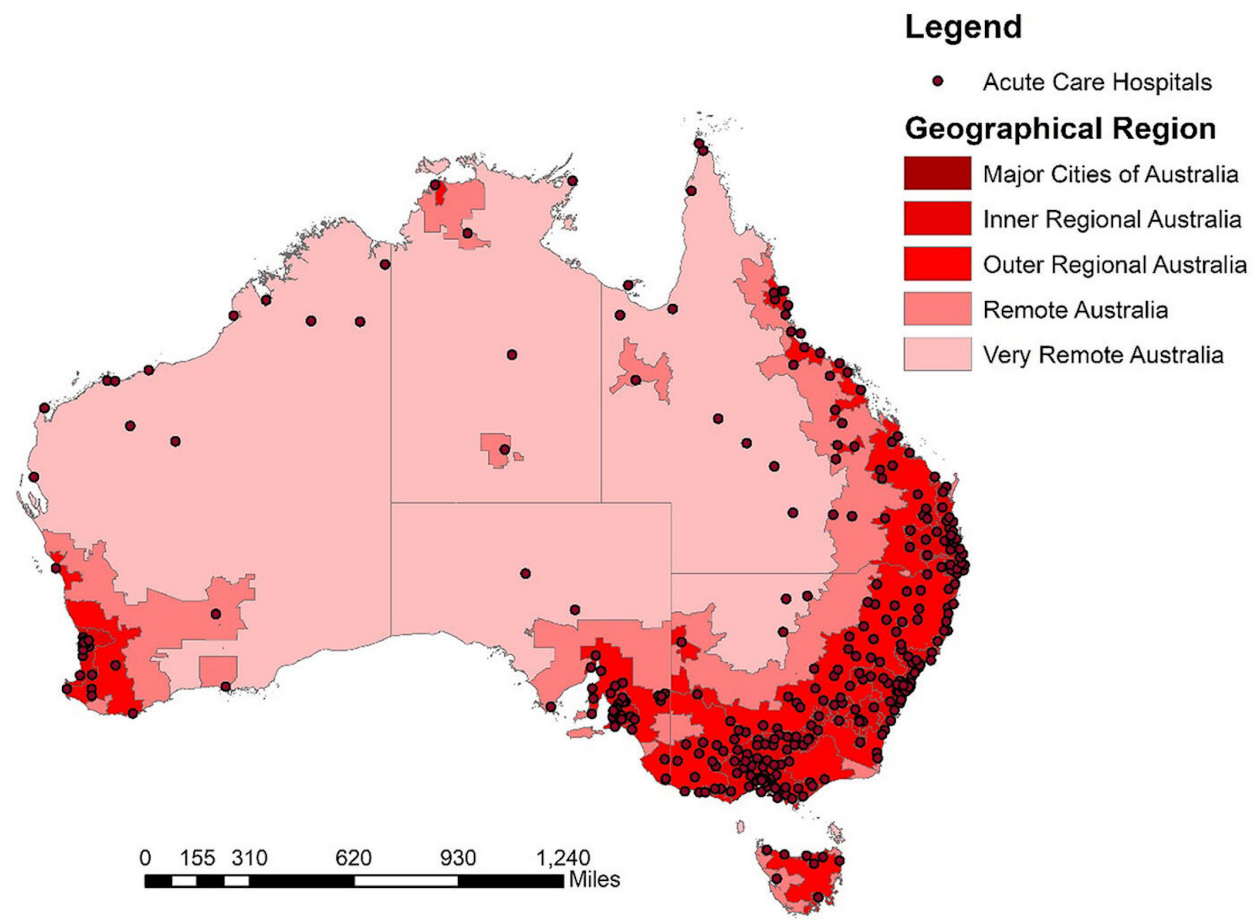

Figure 1 Geographical distribution of hospitals throughout Australia. Approximately $70 \%$ of the Australian population are in densely populated major cities with the remainder distributed through sparsely populated regional and remote areas. There are more than 1322 public and private hospitals distributed throughout eight states and territories. Public hospitals that predominately provide acute care are indicated in the figure. Geographical region is based on the Australian Standard Geographical Classification.

USA, UK, Australia, New Zealand and Canada suggesting $9.2 \%$ of hospitalised patients suffer an adverse event, with nearly half $(43.5 \%)$ of these events deemed preventable, despite these countries having advanced healthcare systems. Many of these adverse events occur after hospital discharge, for example, about $20 \%$ of patients experience an adverse event in the 1st month post-discharge, ${ }^{3}$ a rate that is almost double the reported rate of in-hospital adverse events. Large-scale population-wide studies, predominantly from North America, also suggest high rates of death and unplanned readmissions following hospitalisations, ${ }^{4}$ with these adverse outcomes being a major cause of avoidable healthcare costs. ${ }^{56}$ Moreover, these studies also show that patient outcomes vary two fold to three fold among hospitals for many common conditions, ${ }^{478}$ suggesting variations in quality of care. ${ }^{47}$

\section{Healthcare outcomes in Australia}

In Australia, far less is known about these harms because post-discharge outcomes of care are not systematically captured or analysed. More than 10 million hospitalisations occur annually in Australia for the treatment of a range of conditions across 1322 structurally diverse, and geographically dispersed, public and private hospitals (Figure 1) at a cost of $>\$ A 60$ billion/year. ${ }^{9}$ The Quality in Australian Health Care Study, performed nearly two decades ago, found $16.6 \%$ of patients experienced an adverse event leading to disability or a longer hospital stay with half $(51 \%)$ deemed preventable. ${ }^{10}$ The disability in $13.7 \%$ patients was permanent and $4.9 \%$ of the patients died from a potentially preventable cause. Multiple subsequent studies show a persisting $6.9 \%-16.6 \%^{9-11}$ rates of adverse events among Australian hospitals, ${ }^{11}$ suggesting suboptimal patient safety. However, there are no largescale national studies of post-discharge adverse events, deaths or unplanned readmissions in Australia. The lack of post-discharge outcome data in Australia means that adverse outcomes of hospital care, and the full impact of these events on patients and the health system, are substantially underestimated. The main barriers have been accessing hospitalisation data and the inability to link individual patient data across hospitals and with time to measure post-discharge outcomes on a national scale.

Direct healthcare costs associated with these adverse outcomes are also uncertain. In 2016-2017 healthcare cost Australia over \$A112.0 billion, with $47.8 \%$ (\$A63.8 billion) of the total cost spent on public hospitals, making hospitals the largest area of health expenditure. ${ }^{12}$ Preventable in-hospital complications alone are estimated to contribute $\$ \mathrm{~A} 1.5$ billion to this cost. ${ }^{13}$ However, little or no information currently exists about the resource impact of post-discharge outcomes on the Australian health system, meaning that the impact of adverse events and related avoidable healthcare costs are likely to be underestimated. For example, the cost of unplanned readmissions within 30 days in the US Medicare population has been estimated at US $\$ 17$ billion per year, ${ }^{6}$ much of which is avoidable expenditure as many of these readmissions are preventable. ${ }^{14}$ Cost and resource 
considerations are major drivers of decision-making for clinicians, health services and government. Compressively quantifying avoidable healthcare expenditure in the Australian setting may, therefore, provide a strong catalyst for change, and may assist the development of cost-effective interventions to improve patient outcomes.

Public concerns also exist about unwarranted variation in processes of care among Australia hospitals although there are only a few studies that have examined variation in outcomes. ${ }^{11} 1516$ While these studies focus on a limited number of conditions, they consistently show marked variation in use of treatments or procedures among hospitals which are not explained by variations in patient demographics, suggesting disparities in care. The Australian Productivity Commission, ${ }^{17}$ an independent policy advisory body on a range of issues affecting the welfare of Australians, recommended reporting on basic outcome measures such as mortality and readmissions to inform patients and build greater transparency and accountability, citing significant opportunities to improve healthcare efficiency. Similarly, the Australian Commission on Safety and Quality in Health Care (ACSQHC), has produced the Atlas of Healthcare Variation which has shown substantial geographical variation in use of healthcare across Australia. ${ }^{18}$ The commission has further proposed to report outcomes of care across public and private hospitals and make this information available to the public. Profiling hospital variations in outcomes allows rapid detection of 'outlier' hospitals with high adverse event rates, thereby providing a mechanism to rapidly intervene and minimise potential safety and quality problems. These methods can facilitate local quality improvement efforts by feedback of data to stimulate hospitals to critically examine their outcomes, and when necessary, invest in infrastructure, protocols and other strategies to reduce adverse outcome rates over time. Lastly, profiling variation in outcomes promotes knowledge translation from positive deviation that is, learning from facilities with low adverse outcome rates to identify innovative strategies to improve care.

\section{SAFER Hospitals: a national data linkage study}

SAFety, Effectiveness of care and Resource use among Australian Hospitals (SAFER Hospitals) is a nationwide cohort study that, for the first time in Australia, brings together linked hospitalisation and outcome data from all Australian states and territories in addressing these knowledge gaps. Every Australian hospital collects data on all hospital encounters using a nationally standardised set of data definitions. Individual records can be linked within the datasets, and with others such as death registries, making it feasible to track important outcomes after hospitalisations.

The SAFER Hospitals study will create a national data collection to estimate hospital-wide incidence of outcomes of hospital-based care across all conditions, separating hospital-wide effects on patient outcomes from conditionspecific effects. It will also assess the variation in these outcomes among hospitals, quantify the consequences of adverse outcomes on patients in the short-term and longterm, and assess their impact on healthcare systems with a focus on potentially avoidable health system costs and resources used.

\section{The specific study aims are to}

1. Assess the safety of hospital care by estimating the incidence of serious adverse events and consequences of these adverse events on short-term and long-term patient outcomes.

2. Assess the effectiveness of hospital care by (a) estimating the incidence of rehospitalisation (inpatient readmissions and emergency care encounters) and allcause mortality post-hospitalisation; and by (b) quantifying the proportion of these events that result from potentially preventable causes.

3. Assess healthcare costs and resource use associated with adverse outcomes defined in aims 1 and 2, and to estimate the proportion of costs and resources that may be avoidable.

4. Compare variation in serious adverse events, deaths and unplanned readmissions among hospitals using standardised methods that account for differences in hospitals' case-mix and volume.

5. Develop and test the application of advanced computational methods such as machine learning in facilitating more accurate detection and prediction of adverse outcomes.

6. Prospectively evaluate the temporal change in specific outcomes of hospital care by periodically re-evaluating these outcomes until 2030.

\section{METHODS AND ANALYSIS \\ Study design}

A population-wide cohort study consisting of retrospective and prospective components, using routinely collected administrative data from all public and most $(86 \%)$ private hospitals in Australia. Private hospital data are not available to researchers from South Australia, Northern Territory and Tasmania which collectively contain about $14 \%$ of all private hospitals in Australia. ${ }^{19}$ Figure 2 outlines the study schema.

\section{Study cohort}

We include all hospitalised patients aged $\geq 18$ years from each state and territory's Admitted Patient Collections (APC) from 1 January 2012 to 31 December 2018. Each APC records all inpatient and same-day admissions from all public and most private sector hospitals and day procedure centres. Patient data are collected using a standardised set of variables defined by the National Hospital Minimum Data set for admitted patient care. ${ }^{20}$ The information collected in each APC includes patient sociodemographic characteristics, geographical region, source of referral to the service, acute and elective status of the encounter and service referred to on separation, primary and up to 50 secondary diagnoses and procedures, 


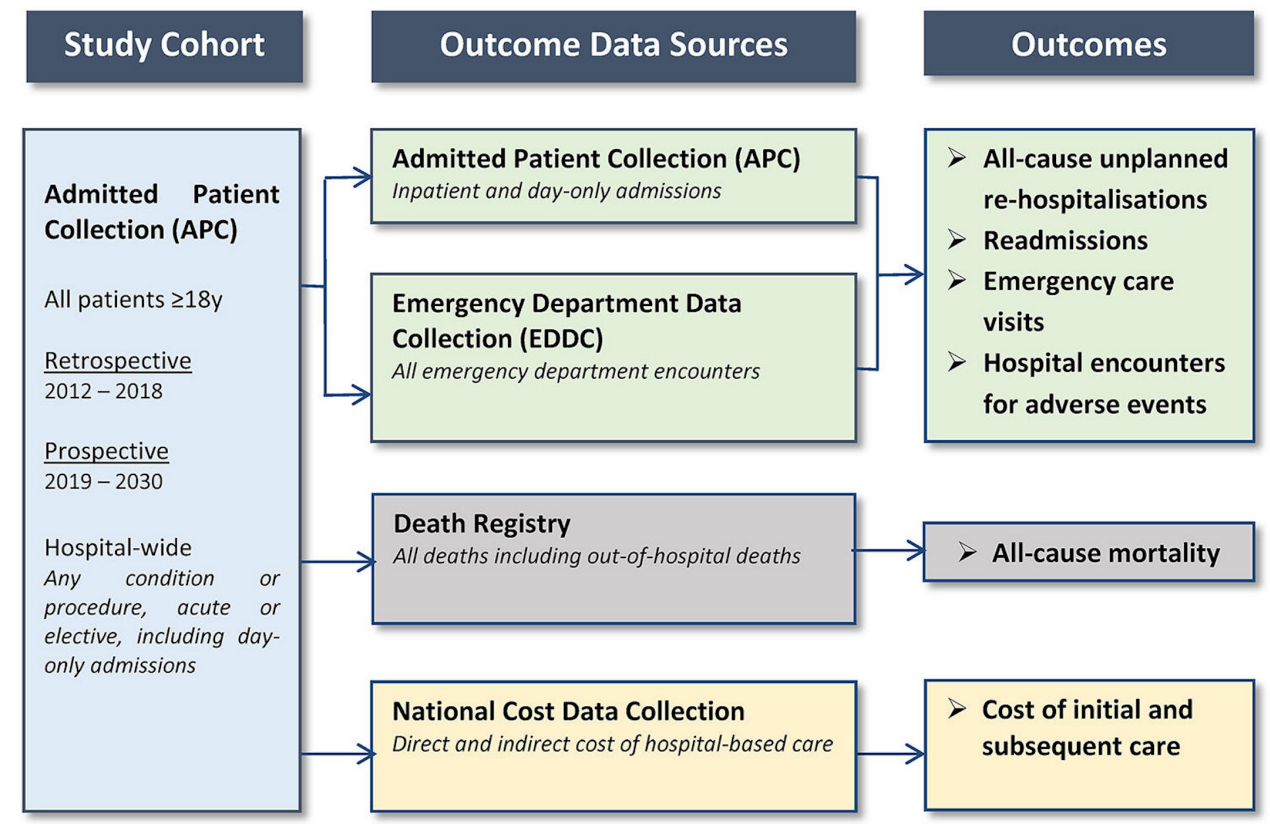

Figure 2 Study schema of the SAFety, Effectiveness of care and Resource use among Australian Hospitals cohort, data sources and outcomes. The study cohort is selected from the Admitted Patient Collections of each state and then linked to subsequent data sources to measure outcomes.

external causes of injury and patient status at discharge. All diagnosis data are coded per International Classification of Diseases and Related Problems, 10th revision Australian Modification (ICD-10-AM) and procedures are coded per the Australian Classification of Health Interventions. Validation audits against medical records have shown relatively good $(>85 \%)$ accuracy of coded data. ${ }^{21}$

From the APCs, we include all hospitalised patients irrespective of condition, as the primary objective is to study hospital-wide outcomes (ie, across all conditions). Adverse outcomes of hospital care are frequently driven by broad hospital-wide phenomenon such as hospital quality control systems and discharge practices in addition to model of care factors related to the underlying condition. Consistent with prior population studies, ${ }^{11} 22$ this approach allows evaluation of hospital-wide phenomenon separate from condition specific effects. Examining hospital-wide outcomes at the onset also enables a 'topdown' approach to subsequently examining conditionspecific outcomes. To facilitate this, we will examine outcomes by the 23 Major Diagnostic Categories (MDCs) into which all patient diagnoses fall, with MDCs generally corresponding to the major organ systems of the body.

\section{Patient comorbidities}

Patient comorbidities are identified using the Condition Category (CC) clinical classification that groups ICD codes into clinically meaningful conditions using diagnosis and procedure codes from the index admission and from any hospitalisations in the preceding 12 months. We use the CC classification because it is widely used to derive comorbidities from routinely collected hospital data. ${ }^{23}$ While the CC model uses ICD-9-CM coding, we have developed an equivalent model based on ICD-10-AM coding for use with Australian data. ${ }^{24}$

\section{Study outcomes}

The primary study outcomes are adverse events, allcause mortality, all-cause unplanned rehospitalisations (including unplanned inpatient readmissions and emergency care encounters) and measures of healthcare costs occurring in hospital and after discharge. The study design enables short-term and long-term outcome assessment.

\section{Adverse events}

We define adverse events using the Classification of Hospital Acquired Diagnoses (CHADx) developed by ACSQHC using ICD-10-AM diagnosis codes. Adverse events are identified from the APC using these diagnosis codes and the condition onset flag to indicate that the adverse event occurred in hospital and was not a preexisting condition prior to hospitalisation. The CHADx taxonomy can be further condensed to form a list of 16 hospital-acquired conditions which identify the more common and serious adverse events that can be accurately measured using routinely collected data. This was developed through a comprehensive process that included literature review, clinical engagement and validation using clinical data.

\section{All-cause mortality}

All deaths are determined by linking APC cohort records with each jurisdiction's Registry of Deaths which record the cause and date of death including out-of-hospital deaths. Death occurring in hospital and within the 
emergency department is recorded within the APC and the Emergency Department Data Collection (EDDC, see below), respectively.

\section{All-cause unplanned rehospitalisations}

All-cause rehospitalisations are defined as the composite outcome of inpatient readmissions and emergency department presentations. ${ }^{614}$ Readmissions to any hospital after the index hospitalisation are measured by linking the study cohort records to subsequent records within each region's APC. Emergency care visits will be assessed by linking hospitalisation records with each region's EDDC. EDDC records all emergency department (ED) encounters using a standardised set of variables from all public and selected private hospitals with ED facilities, including source of referral, mode of presentation, triage category, primary ED diagnosis and the service referred to on separation.

We only count unplanned readmissions in the outcome of rehospitalisation as planned (elective) admissions for scheduled care are a part of normal care and are less likely to be related to care quality. We removed planned admissions from the outcome using the 'Care type' and 'Acute/elective' admission status variables that identify acute care from non-acute and subacute encounters. Since emergency care is reserved for acute presentations, all emergency care visits are regarded as unplanned.

\section{Direct costs and healthcare resource use}

Direct costs associated with hospital care is estimated using publicly available summary cost data from the National Hospital Cost Data Collection (NHCDC) ${ }^{25}$ The NHCDC collates patient-level cost data from $>400$ public and private hospitals in Australia using standardised definitions and contains the average cost associated with hospitalisation for various conditions as defined by the Australian refined diagnostic-related group (DRG, derived from the principal diagnosis and procedures), the proportion of costs that are direct and indirect (overheads) and the components of the cost such as the average amount spent on beds, pharmacy, allied health and pathology. NHCDC also contains the average cost associated with ED encounters as defined by the urgency-related group (URG) for ED presentations. As NHCDC does not publish average DRG/URG costs from private hospitals, averages costs for these hospitals will be calculated using the average cost per DRG or URG of the public hospitals' data in the same year and the ratio of the cost weight per DRG (or URG) between private and public hospitals.

Linkage of patient records within and across datasets is performed within each state or territory except where cross jurisdictional (across state) linkage is readily possible such as between New South Wales and the Australian Capital Territory, and South Australia and Northern Territory. All data linkages will be performed by designated Data Linkage Units within each jurisdiction using probabilistic matching using multiple patient identifiers (such as age, sex, date of birth and Medicare number) with reported accuracy exceeding 99\%. ${ }^{26} 27$ Once linked, de-identified patient data are released to researchers. Data from each jurisdiction are then aggregated to compose a national dataset consisting of equivalent variables from each region. All datasets and linkage components are listed in the online supplemental appendix.

\section{Analysis plan}

Aim 1: Estimate the national incidence of serious adverse events, deaths and unplanned rehospitalisations occurring in hospital and post-discharge.

The incidence of major outcomes of hospital care (adverse events, deaths, unplanned rehospitalisations) is separately estimated by calculating the proportion of all hospitalisations that experienced at least one outcome within 90 days of their initial hospitalisation expressed as a percentage of all hospitalisations. To describe the timing of these outcomes, we will use time-to-event analysis (Kaplan-Meier method and Cox Regression) to generate unadjusted and adjusted event-free survival curves at each time point. In survival models for adverse events and deaths, time will be measured as the number of days from admission until the first occurrence of the outcome. For rehospitalisations, time is measured from discharge in patients who are discharged alive. Patients will also be censored if they do not experience the outcome of interest or reach the end of the 90 day follow-up period. Outcomes will be assessed by geographical region and adjusted for temporal change in baseline characteristics to account for changes in underlying population characteristics over time.

Aim 2: Assess the direct healthcare costs associated with these adverse outcomes and determine the proportion that may be avoidable.

The total cost associated with adverse events is defined as the composite of (1) the incremental cost associated with adverse events occurring in hospital and (2) the cost associated with one or more hospital encounters for adverse events occurring within the 90 days of the index admission. To estimate incremental cost of adverse events occurring in hospital, a generalised linear model with a log-link function and a gamma distribution ${ }^{28}$ will be used, adjusting for differences in baseline characteristics between patients with and without an adverse event. The direct cost of post-discharge adverse events will be estimated by matching the DRG or URG associated with the episode of care to the average cost associated with the DRG or URG published in the NHCDC. For patients with more than one episode during the outcome time frame, costs will be summed to provide a total cost. All costs will be converted to current Australian dollars using health index deflators. Direct costs associated with unplanned rehospitalisations are estimated by matching the DRG or URG associated with the rehospitalisations to the average cost associated with the DRG published in the NHCDC in a similar manner to estimating costs associated with post-discharge adverse events. Costs will be assessed and compared by geographical region. 
To estimate the proportion of readmissions that may be avoidable, we will use a classification system previously used to identify readmissions that are potentially related to hospital care from coded data. While it is challenging to capture the exact reasons for readmissions from coded or clinical data, this classification system provides a reasonable approach in determining the extent to which a readmission is preventable or avoidable. We will classify each readmission into four groups: (1) due to adverse event; (2) for the same diagnosis as the index admission; (3) potentially related to the index diagnosis and (4) other (unrelated) conditions. Group 1 identifies readmissions for hospital-acquired (iatrogenic) conditions such as pulmonary embolus and adverse drug events. This provides a conservative estimate of costs that are most avoidable. To a lesser extent, groups 2 and 3 (readmissions for same or a related condition) also indicate opportunities to reduce cost by optimising patient care, although recognising that it is difficult to distinguish from re-admissions due to disease progression. Group 4 represents cost due to unrelated readmissions, which are the least avoidable. All emergency care visits are deemed unplanned, and therefore avoidable, when estimating costs. We will then sum the total costs in each group representing the proportion of avoidable healthcare costs. Variation in costs will be assessed by geographical region. We will also explore the possibility of combining the four categories of hospital readmissions to create a robust overall index with the weighing of categories to be determined based on empirical testing, available literature and expert clinical opinion.

Aim 3: To detect unwarranted institutional variation in these outcomes among hospitals accounting for case-mix differences which may suggest variation in care quality.

To profile variation in patient outcomes among hospitals, adjusting for differences in case-mix, we estimate each hospital's risk-standardised outcome rate (RSOR) using a hierarchical generalised linear model (HGLM) that accounts for differences in the hospital case-mix, sample-size and clustering of patients within hospitals.

For hospital-wide analyses, there are published riskadjustment models for different outcomes (eg, deaths, readmissions) for different clinical conditions that consider the complexity and diversity of procedures undertaken among hospitals. Such models do not typically estimate risk-standardised rates for one hospitalwide cohort as one model including all admissions would not account for differences in risk variables across different conditions. Instead, the risk models are based on a composite risk adjustment model that includes several subgroups of patients. For example, Horwitz et $a l^{7}$ described the development and use of methods to profile hospital-wide 30-day unplanned readmissions. Here, all admissions are grouped into five cohorts made up of conditions or procedures with relatively similar readmission and post-discharge mortality rates, that are likely to be cared for by similar teams of clinicians and that would generate an adequate sample size for most hospitals. Risk adjustment models are developed within each cohort and a weighted score, based on the distribution among cohorts, is used to estimate an overall model. Similar composite risk-adjustment models are currently being developed for 30-day mortality. ${ }^{29}$ Our goal is to develop similar approaches to risk-adjustment for each outcome using Australian data.

The hospital specific RSOR is calculated as the ratio of predicted hospital outcomes over expected hospital outcome, multiplied by the crude national average outcome rate. The predicted number of outcomes is calculated based on the hospital's case-mix and the estimated hospital-specific intercept term. The expected number of outcomes is calculated based on the hospital's case-mix and national average intercept. The ratio is then multiplied for each hospital by the overall crude outcome rate for ease of interpretation. Bootstrapping with 1000 replications will be used to empirically construct a $95 \%$ CI estimate for each hospital's RSOR using the percentile method. A hospital is deemed a statistical outlier if the hospital's entire 95\% interval estimate is above or below the national average. This approach for estimation of the RSOR ensures that the observed variation among hospitals is not due to underlying differences in case-mix or procedure-mix and is consistent with methods we have previously used for profiling variation in outcomes among hospitals. ${ }^{1530}$ All hospital-level analyses will be limited to unique hospitals with at least 25 hospitalisations during the study period to enable a robust estimate of the hospital outcome rate.

Aim 4: Test machine learning methods to better predict adverse outcomes of hospital care to inform service improvement initiatives.

This aim will test the accuracy of machine learning methods, compared with conventional regression models, for predicting adverse outcomes such as death and readmissions which may be useful in developing automated methods that identify high-risk individuals and facilitate service improvement. Machine learning, a subfield of artificial intelligence, refers to an array of data-driven automated analytical techniques that rely on sophisticated pattern-recognition methods, which can 'learn' from large-scale datasets. Machine learning is widely used in industry for rapid automated analysis of massive volumes of routinely collected data such as that collected in hospitals in the process of delivering care. While machine learning methods initially failed to suggest superiority over conventional statistical methods, more recent analyses suggest significantly better ability to predict outcomes from administrative datasets. ${ }^{31}{ }^{32}$ To achieve this goal, we will develop machine learning methods including deep learning that can automatically extract relevant features from data collected from hospitals. We will train models using a five-fold cross validation experiment, where for each of the folds, a random set containing $80 \%$ of the dataset samples will be used for training and the remaining $20 \%$ will then be used for testing the model. All models will be compared with 
a standard logistic regression model using the model c-statistic.

Aim 5: To prospectively evaluate the temporal change in each of the outcomes of hospital care by periodically re-evaluating these outcomes until 2030.

This prospective evaluation will assess how outcomes change with time at both the patient and hospital level. This aim will be achieved by prospectively extending the cohort every 4 years from 2019 until 2030 and comparing the longitudinal trend in each outcome using standardised methods. The Cochran-Armitage trend test or HGLM will be used to assess for statistically significant trends over time. Finally, the study results will be reported in accordance with the Strengthening the Reporting of Observational Studies in Epidemiology statement. ${ }^{33}$

\section{Supplementary analyses}

In most regions, de-identified data are provided for analysis including de-identified hospital labels where hospital names and unique identifiers are replaced with a dummy identifier so that hospital-level analyses are possible, but the hospital cannot be identified. In New South Wales, Queensland and Tasmania, actual hospital identifiers are provided. In these regions, we will seek to further examine the association of hospital-specific characteristics with the outcomes using publicly available data from the National Public Hospital Establishments Database (NPHED) ${ }^{34}$ and the MyHospitals ${ }^{35}$ reporting portal. Using standardised data definitions, NPHED collects data on each hospital's revenue, staffing levels, expenditure, the number of available beds for admitted patients, geographical location, specialised service indicators and the type of non-admitted patient care. ${ }^{34}$ NPHED data are only available for public hospitals. The MyHospitals national reporting platform allows users to explore information about hospitals and publicly reports a selected number of hospital-specific performance process and outcome indicators such as healthcare-associated infection rates, predominately from public hospitals. ${ }^{35}$ New South Wales, Queensland and Tasmania include 54\% of the Australian population and New South Wales and Queensland encompass $52.8 \%$ of all Australian hospital beds. Hospital bed numbers in Tasmania are not publicly released to protect the confidentiality of the small number of private hospitals in Tasmania.

\section{Patient and public involvement}

No patient involved.

\section{Ethical approval}

Human Research Ethics Committees (HRECs) of all Australian states and territories provided ethical approval for the conduct of the study. Specifically, approval was provided by the New South Wales Population \& Health Services Research Ethics Committee (ref\# HREC/17/ CIPHS/58), The Australian Capital Territory Health Department HREC (ref\# ETH.4.18.073E), Calvary Health Care Human Research Ethics Committee (ref\#
24-2018), Human Research Ethics Committee of the Northern Territory Department of Health (ref\# 20183183), Western Australian Department of Health HREC (ref\# 2018/25) and the Tasmanian Department of Health HREC (ref\# H0017507). States of Queensland, South Australia and Victoria accepted NSW HREC approval through a National Mutual HREC Acceptance Scheme. All HRECs granted a waiver of informed consent for the use of de-identified patient data.

\section{Privacy and data security}

De-identified study data will be securely stored at the Basil Hetzel Institute for Translational Research. A copy of the study data will be stored at the Secure Unified Research Environment (SURE). SURE is a highly secure, remote access data storage and analytic facility specifically designed for storage and analysis of large volumes of healthcare data. ${ }^{36}$ It uses extensive measures to ensure data security including data encryption, secure firewalls, password protection, logging of access to files and a 'curated gateway' preventing data from being copied or moved out of the secure environment. Use of SURE facilitates secure access to study data for study investigators located throughout Australia.

\section{Dissemination and translation}

The study team involves stakeholder representatives and the study investigators are in advisory roles in safety and quality organisations such as the Quality and Safety Committee of the Royal Australasian College of Physicians, Australian Patient Safety Foundation and ACSQHC. As the study progresses, the investigators will seek further engagement with state and federal stakeholders and professional bodies to facilitate dissemination and translation. We will also establish an independent Study Advisory Panel which will include stakeholders' involvement. This panel will meet two times a year and provide independent study advice and guidance. This provides a format for continued engagement of stakeholders as the study progresses, gains buy-in and creates a mechanism for the investigators to address stakeholder priorities and concerns. The findings will also be disseminated through scientific publications and briefing documents. Plain language summaries and infographics will also be used to promote research outcomes to the wider community via media releases and social media.

\section{DISCUSSION}

In Australia, like other countries with comparable highly developed healthcare systems, there are concerns about the safety and quality of, and potential variations in, hospital care. Harms associated with hospital care are poorly reported, partly because of the inability to capture adverse outcomes after discharge. Consequently, there are no national studies of post-discharge adverse events, deaths or readmissions. The avoidable adverse outcomes and associated healthcare costs are uncertain and 
whether these vary among hospitals, suggesting variation in care quality, is unknown. The SAFER Hospitals study purports to answer these questions and benefit the Australian community by (1) informing and prioritising target conditions for large-scale quality improvement efforts; (2) developing and supporting the implementation of standardised methods for hospitals to routinely measure these outcomes and (3) facilitating policy changes such as public reporting efforts and innovative funding models to incentivise safer and more effective care. National studies of adverse outcomes are largely limited to North America and there is considerable interest in the generalisability of these outcomes to other health systems. Therefore, the outcomes of this study are likely to have broad relevance to the international literature.

This study has important limitations that need consideration. The study data are linked only within each state and territory, except for cross-jurisdictional linkage between the state of New South Wales and Australian Capital Territory, and between South Australia and Northern Territory. While this may limit the ability to track outcomes for patients who are transferred across state borders, prior studies show that such transfers involve $<3 \%$ of all patients and hence the likely impact on our outcome measurements will be small. ${ }^{37}$ Moreover, we limit our analyses to patients residents within each jurisdiction and exclude out-of-state residents to further minimise potential error. Our study is based on routinely collected administrative data which is less granular and potentially less accurate than data collected specifically for clinical research or quality registries. While well-developed standards for coding of clinical diagnoses exist, retrospective coding of diagnoses depends on the diagnoses being recorded in the patient's medical record, rather than based on the prospective application of external criteria, as would occur in a clinical trial. Routinely collected hospitalisation data also do not capture in-hospital medication use or the results of investigations such as pathology results. Nevertheless, validation studies have shown relatively good accuracy of diagnoses and procedures coding within administrative data compared with medical records. ${ }^{21}$ Linking routinely collected data is also the most accurate method for tracking post-discharge outcomes of care. It is not feasible to collect detailed individual patient data at a national scale and thus an observational study using routinely collected data is the suitable data source for this purpose. South Australia, Tasmania and the Northern Territory do not release private hospital data to researchers although the impact on the study is likely to be small as most acute care in Australia is provided by public hospitals. These states only account for about $10 \%$ of the overall population. Furthermore, private hospitals in these states encompass a small proportion of overall hospital beds. For example, those in South Australia form $2 \%$ of all hospital beds in Australia and the number of private hospital beds in Northern Territory and Tasmania is not publicly released to protect the confidentiality of the small number of private hospitals in these regions. ${ }^{19}$ Socioeconomic status may influence patient outcomes although socioeconomic characteristics are not routinely collected in the hospital Admitted Patient Data Collections.

\section{Author affiliations}

${ }^{1}$ Department of Cardiology, The Prince Charles Hospital, Brisbane, Queensland, Australia

${ }^{2}$ School of Clinical Medicine, The University of Queensland, Brisbane, Queensland, Australia

${ }^{3}$ Faculty of Health and Medical Sciences, The University of Adelaide, Adelaide, South Australia, Australia

${ }^{4}$ College of Medicine and Public Health, Flinders University, Adelaide, South Australia, Australia

${ }^{5}$ Australian Commission on Safety and Quality in Health Care, Sydney, New South Wales, Australia

${ }^{6}$ Healthcare Quality Intelligence Unit, Patient Safety and Clinical Quality Directorate, Clinical Excellence Division, Department of Health Government of Western Australia, Perth, Western Australia, Australia

${ }^{7}$ Faculty of Engineering Computer and Mathematical Sciences, The University of Adelaide, Adelaide, South Australia, Australia

${ }^{8}$ George Institute for Global Health, Sydney, New South Wales, Australia ${ }^{9}$ Concord Repatriation General Hospital, Sydney, New South Wales, Australia

${ }^{10}$ Menzies School of Health Research, Casuarina, Northern Territory, Australia

${ }^{11}$ Health Economics Unit, Flinders University, Adelaide, South Australia, Australia

${ }^{12}$ The Prince of Wales Hospital, Sydney, New South Wales, Australia

${ }^{13}$ Intensive Care Services, Central Adelaide Local Health Network, Adelaide, South Australia, Australia

${ }^{14}$ Internal Medicine and Clinical Epidemiology, Princess Alexandra Hospital, Brisbane, Queensland, Australia

${ }^{15}$ Adelaide Geriatrics Training and Research with Aged Care (GTRAC) Centre, The University of Adelaide, Adelaide, South Australia, Australia

${ }^{16}$ Aged \& Extended Care Services, The Basil Hetzel Institute, The Queen Elizabeth Hospital, Adelaide, South Australia, Australia

${ }^{17}$ Flinders Centre for Epidemiology and Biostatistics, Flinders University, Adelaide, South Australia, Australia

Acknowledgements The SAFER Hospitals investigators would like to thank the following Data Linkage Units and responsible individuals for their assistance and considerable in-kind support provided. New South Wales \& Australian Capital Territory: Centre for Health Record Linkage; South Australia and Northern Territory: SA-NT DataLink; Queensland: Statistical Services Branch, Queensland Department of Health; Tasmania: Tasmanian Data Linkage Unit; Victoria: Centre for Victorian Data Linkages, Victorian Department of Health; Western Australia: Data Linkage Branch, Western Australian Department of Health.

Contributors IR conceived the study, developed the study design and drafted the manuscript. IR, SH, AA and DH assisted in the study design, data acquisition and drafting of the manuscript. RA, GC, MG, SG, IS, and RV contributed to the study design. RA, BA, CB, GC, MG, SG, BK, SK, GO, IS, RV and RW contributed to critical revision of the manuscript. All authors approved the final version to be published. All authors agree to be accountable for all aspects of the work.

Funding The Hospital Research Foundation.

Map disclaimer The depiction of boundaries on this map does not imply the expression of any opinion whatsoever on the part of BMJ (or any member of its group) concerning the legal status of any country, territory, jurisdiction or area or of its authorities. This map is provided without any warranty of any kind, either expressed or implied.

Competing interests None declared.

Patient and public involvement Patients and/or the public were not involved in the design, or conduct, or reporting, or dissemination plans of this research.

Patient consent for publication Not required.

Provenance and peer review Not commissioned; externally peer reviewed.

Open access This is an open access article distributed in accordance with the Creative Commons Attribution Non Commercial (CC BY-NC 4.0) license, which permits others to distribute, remix, adapt, build upon this work non-commercially, and license their derivative works on different terms, provided the original work is 
properly cited, appropriate credit is given, any changes made indicated, and the use is non-commercial. See: http://creativecommons.org/licenses/by-nc/4.0/.

\section{ORCID iDs}

Isuru Ranasinghe http://orcid.org/0000-0003-0982-1561

lan A Scott http://orcid.org/0000-0002-7596-0837

\section{REFERENCES}

1 Institute of MedicineKohn LT, Corrigan JM, Donaldson MS, et al, eds. To err is human: building a safer health system, 2000.

2 de Vries EN, Ramrattan MA, Smorenburg SM, et al. The incidence and nature of in-hospital adverse events: a systematic review. Qual Saf Health Care 2008;17:216-23.

3 Forster AJ, Murff HJ, Peterson JF, et al. The incidence and severity of adverse events affecting patients after discharge from the hospital. Ann Intern Med 2003;138:161-7.

4 Ranasinghe I, Parzynski CS, Searfoss R, et al. Hospital Visits after Hospital Outpatient Surgery Measure: Risk-Standardized Hospital Visits within 7 Days After Hospital Outpatient Surgery Measure. In: Yale new Haven health services Corporation - center for outcomes research and evaluation (YNHHSC/CORE. Centers for Medicare \& Medicaid Services (CMS), 2016.

5 HealthGrades. The eighth annual HealthGrades patient safety in American hospitals study 2011.

6 Jencks SF, Williams MV, Coleman EA. Rehospitalizations among patients in the Medicare fee-for-service program. N Engl $J$ Med 2009;360:1418-28.

7 Horwitz LI, Partovian C, Lin Z, et al. Development and use of an administrative claims measure for profiling hospital-wide performance on 30-day unplanned readmission. Ann Intern Med 2014;161:S66-75.

8 Tsai TC, Joynt KE, Orav EJ, et al. Variation in surgical-readmission rates and quality of hospital care. N Engl J Med 2013;369:1134-42.

9 Australian Institute of Health and Welfare. Admitted patient care 2014-15: Australian hospital statistics. health services series no 68 cat no HSE 172. Canberra: Australian Institute of Health and Welfare, 2016.

10 Wilson RM, Harrison BT, Gibberd RW, et al. An analysis of the causes of adverse events from the quality in Australian health care study. Med J Aust 1999;170:411-5.

11 Duckett S, Jorm C, Danks L, et al. All complications should count: using our data to make hospitals safer. Grattan Institute: Grattan Institute Report, 2018.

12 Productivity Commission. Report on government services 2019: public hospitals: productivity Commission, 2019. Available: https:// www.pc.gov.au/research/ongoing/report-on-government-services/ 2019/health

13 Duckett SJ, Christine J. Safer care saves money Melbourne. Victoria: Grattan Institute, 2018. https://grattan.edu.au/wp-content/uploads/ 2018/08/Safer-care-saves-money.pdf

14 van Walraven C, Bennett C, Jennings A, et al. Proportion of hospital readmissions deemed avoidable: a systematic review. CMAJ 2011;183:E391-402.

15 Ranasinghe I, Labrosciano C, Horton D, et al. Institutional variation in quality of cardiovascular implantable electronic device implantation: a cohort study. Ann Intern Med 2019;171:309-17.

16 Bureau of Health Information. The insights series - exploring clinical variation in mortality following hospitalisation, seven clinical conditions NSW, July 2012 - June 2015. Sydney (NSW); 2017.
17 Productivity Commission. Efficiency in health. Canberra: Commission Research Paper, 2015.

18 Australian Commission on Safety and Quality in Health Care. Australian atlas of healthcare variation series, 2018. Available: https://www.safetyandquality.gov.au/publications-and-resources/ australian-atlas-healthcare-variation-series\#third-atlas-published2018

19 Australian Institute of Health and Welfare. Hospital resources 2014-15: Australian hospital statistics. In: . Canberra: AlHW., ed. Health services series no 71 Cat no HSE 176, 2016.

20 Australian Institute of Health and Welfare. Admitted patient care national minimum dataset 2016-17. Available: https://meteor.aihw. gov.au/content/index.phtml/itemld/612171 [Accessed 15/02/2019].

21 Henderson T, Shepheard J, Sundararajan V. Quality of diagnosis and procedure coding in ICD-10 administrative data. Med Care 2006;44:1011-9.

22 Wilson RM, Runciman WB, Gibberd RW, et al. The quality in Australian health care study. Med J Aust 1995;163:458-71.

23 Pope GC, Ellis RP, Ash AS, et al. Diagnostic cost group hierarchical condition category models for Medicare risk adjustment. Health Economics Research, Inc Waltham, MA 2000.

24 Ranasinghe I, Barzi F, Brieger D, et al. Long-Term mortality following interhospital transfer for acute myocardial infarction. Heart 2015;101:1032-40

25 The Independent Hospital Pricing Authority. National Hospital cost data collection report: public sector, round 21 financial year 2016-17 2019.

26 Holman CD, Bass AJ, Rouse IL, et al. Population-Based linkage of health records in Western Australia: development of a health services research linked database. Aust N Z J Public Health 1999;23:453-9.

27 Boyd JH, Randall SM, Ferrante AM, et al. Accuracy and completeness of patient pathways--the benefits of national data linkage in Australia. BMC Health Serv Res 2015;15:312.

28 Blough DK, Madden CW, Hornbrook MC. Modeling risk using generalized linear models. J Health Econ 1999;18:153-71.

29 Centers for Medicare \& Medicaid Services (CMS). Hospital-Wide (AllCondition, All-Procedure) Risk-Standardized mortality measure: draft measure methodology for interim public Comment; 2016.

30 Normand S-LT, Shahian DM. Statistical and clinical aspects of hospital outcomes profiling. Statist. Sci. 2007;22:206-26.

31 Khera RHJ, Hurley N, et al. Machine-learning to improve prediction of mortality following acute myocardial infarction: an assessment in the NCDR-Chest Pain-Myocardial infarction registry. bioRxiv 2019.

32 Jamei M, Nisnevich A, Wetchler E, et al. Predicting all-cause risk of 30-day Hospital readmission using artificial neural networks. PLoS One 2017;12:e0181173.

33 Vandenbroucke JPet al. Strengthening the reporting of observational studies in epidemiology (STROBE): explanation and elaboration. Ann Intern Med 2007;147:W.

34 Australian Institute of Health and Welfare. The National public hospital establishments database. Available: https://www.aihw.gov. au/about-our-data/our-data-collections/national-hospitals-datacollection [Accessed 28 Mar 2020].

35 Australian Institute of Health and Welfare. MyHospitals, 2020. Available: https://www.aihw.gov.au/myhospitals [Accessed $28 \mathrm{Mar}$ 2020].

36 Ebah L, Hanumapura P, Waring D, et al. A multifaceted quality improvement programme to improve acute kidney injury care and outcomes in a large teaching hospital. BMJ Qual Improv Rep 2017;6. doi:10.1136/bmjquality.u219176.w7476.

37 Spilsbury K, Rosman D, Alan J, et al. Cross border Hospital use: analysis using data linkage across four Australian states. Med J Aust 2015;202:582-6. 